\title{
Pénalisation de la circulation et reconfigurations de la frontière : le maintien des étrangers en « zone d'attente »
}

Penalizing movement, reframing borders: alien detention in the French "waiting zone"

Penalización de la circulación y reconfiguración de la frontera: el mantenimiento de los extranjeros en "zonas de espera"

\section{Chowra Makaremi}

\section{(2) OpenEdition}

\section{Journals}

\section{Édition électronique}

URL : http://journals.openedition.org/conflits/16133

DOI : $10.4000 /$ conflits. 16133

ISSN : $1777-5345$

Éditeur :

CCLS - Centre d'études sur les conflits lilberté et sécurité, L'Harmattan

Édition imprimée

Date de publication : 30 octobre 2008

Pagination : 55-73

ISBN : 978-2-296-06624-3

ISSN : 1157-996X

Référence électronique

Chowra Makaremi, «Pénalisation de la circulation et reconfigurations de la frontière : le maintien des étrangers en « zone d'attente » », Cultures \& Conflits [En ligne], 71 | automne 2008, mis en ligne le 02 février 2009, consulté le 30 mars 2021. URL : http://journals.openedition.org/conflits/16133; DOI : https://doi.org/10.4000/conflits.16133 


\section{Pénalisation de la circulation et reconfigurations de la frontière : le maintien des étrangers en « zone d'attente»}

\section{Chowra MAKAREMI}

Chowra Makaremi est doctorante en anthropologie à l'université de Montréal. Ses recherches portent sur la détention frontalière en France. Elle a notamment publié "Alien Confinement in Europe: Violence and the Law. The Case of Roissy-Charles de Gaulle Airport in France", in Hogan C., Marín Dòmine M., The Camp: Narratives of Internment and Exclusion, Newcastle, Cambridge Scholars Publishing, 2007 (pp. 39-54).

T es déplacements de populations sont au fondement d'une évolution des idenLtités et des modes d'identification. Ils font l'objet d'une gestion politique fondée sur un rapport d'exclusion autour de lignes de fracture Nord-Sud et sur l'affirmation d'un contrôle étatique de la circulation. Les travaux qui posent les bases d'une telle approche ${ }^{1}$ insistent sur l'importance des modalités du déplacement comme un lieu d'observation privilégié de la réalité globale. Dans les pays occidentaux, le contrôle des frontières et la construction des « camps d'étrangers 2 » témoignent de nouvelles distributions du pouvoir qui passent par l'accès à la mobilité. Le contrôle étatique des mouvements volontaires et forcés de populations s'impose donc à la fois comme un enjeu de gouvernement dans le monde contemporain et comme un angle d'approche pour en comprendre les évolutions essentielles, tel que le lien entre identité, nation et territoire. C'est autour des populations en déplacement que s'énoncent en effet certaines interrogations poli-

1. On peut citer Appadurai A., Après le colonialisme : les conséquences culturelles de la globalisation, Paris, Payot, 2001 ; Bayart J.-F., Le Gouvernement du monde. Une critique politique de la globalisation, Paris, Le Seuil, 2004 ; Agier M., Au bord du monde - Les réfugiés, Paris, Flammarion, 2002 ; Castles S., Globalization and Ethnicity: From Migrant Workers to Transnational Citizens, London, Sage, 2000; Gungwu W. (ed.), "Global History and Migrations”, Global History Series, vol. 2., Oxford, Westview Press, 1997 ; Bauman Z., Le Coût humain de la mondialisation, Paris, Hachette Littératures, 1999.

2. Bernardot M., Camps d'étrangers, Broissieux, Ed. du Croquant, 2008. 
tiques actuelles liées à la notion de citoyenneté 3 . D’une part, la gestion des populations étrangères met à nu la construction étatique de la communauté nationale. D'autre part, leurs modes d'existence esquissent des possibilités d'énonciation et de participation politique déconnectées du paradigme territorial, qui invitent à repenser la « localité » dans les conditions globales. Ces évolutions s'articulent notamment autour d'une ambivalence de la frontière, qui fait coexister deux réalités : celle de la déterritorialisation progressive des organisations socio-économiques et celle du renforcement des frontières comme lieu de pratiques administratives d'exclusion visant les individus. C'est ainsi que s'éclaire l'intérêt apparemment contradictoire des sciences sociales pour la remise en cause des liens entre territoires et identités et, en même temps, pour l'étude de la frontière comme espace sociopolitique. En effet, tandis que des travaux consacrés à la «mondialisation » remettent en question les monopoles de l'Etat et du territoire comme axes de gravité de la réflexion politique ${ }^{4}$, les «borderlands " s'imposent comme des sujets d'étude anthropologique, engageant une réflexion sur la nécessité de repenser la séparation spatiale, les liens entre culture et territoire, spatialité et altérité, en particulier à travers une étude empirique renouvelée des espaces frontaliers et des modalités du contrôle des populations qui s'y joue 5 .

La mise au ban ${ }^{6}$ des acteurs de la circulation, dont témoignent les centres fermés aux frontières, est une réalité difficile à saisir : les barrières instituées de l'illégalité, les enjeux de clandestinité, d'anonymat, d'identification font qu'en pratique, ce parcours ne se raconte pas et se dérobe face à l'observation empirique. Le présent article se fonde ainsi sur une recherche empirique dans la «zone d'attente pour personnes en instances» de l'aéroport de Roissy (dite «Zapi 3 »), dans laquelle les étrangers non admis ou demandant l'asile sont détenus pour une durée maximale de vingt jours. Notre enquête ethnographique à l'intérieur du centre de Zapi 37 a été complétée par une série d'entretiens avec des acteurs et des témoins privilégiés du dispositif, ainsi que des personnes admises sur le territoire français après une période plus ou moins longue

3. Gupta A., Ferguson J. (eds.), Culture, Power, Place: Explorations in Critical Anthropology, Durham, Duke University Press, 1997 ; Balibar E., Nous, citoyens d'Europe ? Les frontières, l'Etat, le peuple, Paris, La Découverte, 2001 ; Blom Hansen T., Stepputat F. (eds.), Sovereign Bodies: Citizens, Migrants, and States in the Postcolonial World, Princeton NJ, Princeton University Press, 2005 ; Sharma A., Gupta A. (eds.), The Anthropology of the State, Malden, Blackwell, 2006.

4. Appadurai A., op. cit.; Gupta A., Ferguson J. (eds.), op. cit.

5. Bietlot M., «Du disciplinaire au sécuritaire : de la prison au centre fermé », Multitudes, n¹1, janvier 2003, pp. 57-66; Courau H., Ethnologie de la forme-camp de Sangatte. De l'exception à la régulation, Paris, Ed. des Archives contemporaines, 2007 ; Evans C., "Asylum Seekers and 'Border Panic' in Australia”, Peace Review, vol. 15, n², juin 2003; Dudziak M.L., Volpp L. (eds.), Legal Borderlands: Law and the Construction of American Borders, Baltimore, John Hopkins University Press, 2006.

6. Bigo D., "Detention of Foreigner, States of Exception, and the Social Practices of Control of the Banopticon", in Kumar Rajaram P., Grundy-Warr C. (eds.), Borderscapes: Hidden Geographies and Politics at Territory's Edge, Minneapolis, University of Minesotta Press, 2007.

7. Fondée sur une participation observante dans le cadre d'un engagement bénévole au sein de l'Association nationale d'assistance aux frontières pour les étrangers (Anafé). 
de maintien. En s'appuyant sur les expériences vécues d'enfermement et de circulation qui se tissent autour du maintien en zone d'attente, il s'agit d'approcher la réalité de la frontière, en sondant les lieux par où les individus transitent et les techniques qui mettent en branle leur circulation faite de passages, d'immobilisations et de retours. Cette "errance individuelle 8 », dans la rugosité de ses parcours improbables, nous renseigne sur l'évolution de l'espace public, du rapport au temps et au (non-)lieu qui fondent une modalité inédite de l'inclusion dans l'organisation politique étatique.

Comment le contrôle frontalier fonctionne-t-il au quotidien ? Quelles fonctions garde-t-il dans une perspective à plus long terme de parcours dans le pays d'accueil ? En un mot, comment les évolutions de la frontière configurent-elles la gestion de la circulation ? Nous développerons quelques pistes de réflexion le long de deux axes de recherche qui, ensemble, interrogent la démarcation entre intérieur et extérieur dont est habituellement chargée la frontière. D’une part, les réorganisations aussi bien matérielles que conceptuelles de la mobilité et de la frontière en jeu dans la zone d'attente aéroportuaire voient l'émergence d'un nouvel espace de circulation qui exploite, aux fins du contrôle, les ambiguïtés et les contradictions de l'organisation territoriale internationale. D'autre part, la fonction frontalière se maintient dans l'espace national à travers une construction de la circulation en illégalisme. C'est ce que nous essayerons de saisir en nous intéressant particulièrement aux ressorts techniques et aux effets de gestion qu'offre la pénalisation dans la production frontalière de cette mise au ban.

\section{L'enfermement dans la circulation et le paradoxe des sans-Etats}

A partir du milieu des années 1970, des étrangers demandant l'asile à leur arrivée en France, ou n'étant pas autorisés à entrer sur le territoire français pour des raisons aussi bien administratives (comme la validité du visa ou l'absence de passeport) que discrétionnaires (comme le soupçon d'immigration illégale) - sont détenus pour une durée variable par la police de l'air et des frontières. Selon l'administration française, dans les aéroports internationaux, l'espace compris entre les pistes et les postes de douane n'est en effet pas encore en France : il n'est pas soumis à la loi française et il ne fait pas non plus l'objet d'une réglementation internationale 9 . Dans l'aéroport de Roissy-CDG, à 23 kilomètres de Paris, c'est-à-dire au cour du territoire français, la frontière aérienne que franchissent plus de 56 millions de personnes par an ${ }^{10}$ est ainsi fictivement restituée dans sa dimension marginale de frontière à travers un espace neutralisé. La

8. Augé M., Non-Lieux, introduction à une anthropologie de la surmodernité, Paris, Le Seuil, 1992 , p. 146.

9. Par défaut, il est alors soumis aux obligations internationales de l'Etat relatives à la Convention européenne de sauvegarde des droits de l'Homme et des libertés fondamentales du 4 novembre 1950 et au Pacte international relatif aux droits civils et politiques du 19 novembre 1966. 
continuité territoriale est suspendue en droit par le recours à une « fiction juridique » - technique du droit qui consiste à supposer vraie une chose que l'on sait fausse, en l'occurrence, que la zone internationale de l'aéroport n'est pas encore la France. Or, à partir du milieu des années 1970, l'administration française réinvestit cette fiction d'extraterritorialité pour en faire le cadre de la gestion des étrangers refusés sur le territoire. La police de l'air et des frontières en charge du contrôle à Roissy développe au fil des années certaines pratiques d'administration des étrangers refusés dans la zone internationale, en inscrivant ces pratiques administratives dans la suspension légale qui définit cette zone. En localisant le contrôle à l'intérieur de cette zone pour bénéficier de son (absence de) statut juridique, l'administration française organise, met en place et ajuste progressivement une technique d'enfermement des étrangers dans la frontière, comme gestion durable du paradoxe frontalier en aéroport. Un quotidien de contrôle s'installe dans ce qui était au départ un tour de passe-passe juridique exceptionnel. Ainsi, l'exercice du pouvoir investit-il un écart entre norme et réel (une «fiction juridique ») et le creuse un peu plus en y développant des pratiques quotidiennes, des habitudes, des règles de fonctionnement, des hiérarchies. En 1992, sous la pression d'associations de défense des droits humains, de défense des étrangers et de syndicats - regroupés en une Association nationale d'assistance aux frontières pour les étrangers (Anafé) - et sous la menace de plusieurs procédures judiciaires ${ }^{11}$, le ministre de l'Intérieur socialiste Paul Quilès présente une loi visant à donner un cadre juridique à une pratique administrative en cours, en instituant les «zones d'attente pour personnes en instance » 12 . L'investissement technico-légal de la détention aux frontières répond à la demande de mise en conformité démocratique, en même temps qu'elle solidifie et verrouille le dispositif administratif en place.

Contrairement au contexte dans lequel s'inscrivent la plupart des ethnographies consacrées aux passages frontaliers ${ }^{13}$, la zone d'attente déborde ainsi l'analyse de la frontière en termes de point de contact et de séparation, dans la mesure où elle n'est pas un passage entre deux lieux. Justement, la procédure

10. Source : Aéroports de Paris : http://www.aeroportsdeparis.fr/ExpFiles/RA-ADP-2006\%20\%20FR\%2072.pdf, consulté le 23 septembre 2008.

11. Le 25 mars 1992, le Tribunal de grande instance de Paris condamne le ministère de l'Intérieur, jugeant que l'Etat a "gravement porté atteinte à la liberté » de six demandeurs d'asile en les retenant dans un hôtel, à l'aéroport de Roissy-Charles-de-Gaulle. L'Etat est condamné à payer 33000 francs de dommages et intérêts aux intéressés et 1 franc symbolique au Groupe d'information et de soutien des travailleurs immigrés (GISTI). Cette décision affirme l'illégalité de la «zone internationale », où sont placés les étrangers.

12. Loi n ${ }^{\circ}$ 92-625 du 6 juillet 1992 sur la zone d'attente des ports et des aéroports et portant modification de l'ordonnance $\mathrm{n}^{\circ} 45-2658$ du 2 novembre 1945 relative aux conditions d'entrée et de séjour des étrangers en France, dite " loi Quiles».

13. Donnan H., Wilson T.M., Borders: Frontiers of Identity, Nation and State, Oxford, Berg, 1999; Heyman J., "Putting Power in the Anthropology of Bureaucracy: the Immigration and Naturalization Service at the Mexico-United States Border”, Current Anthropology, n`36, avril 1995 ; Coutin S.B., "Illegality, Borderlands, and the Space of Non Existence", in Perry R.W., Maurer B. (eds.), Globalization Under Construction: Governmentality, Law and Identity, Minneapolis, University of Minnesota Press, 2003. 
de « maintien à la frontière » existe parce qu'il est impossible, dans le cadre de la circulation aérienne, de refouler les étrangers non documentés de l'autre côté de la frontière, comme c'est par exemple le cas aux postes de douane aux frontières routières. La zone d'attente a été créée car il y a n'y pas d' « autre côté ». Elle n'est, dès lors, pas une frontière au sens traditionnel d'un "ordre social qui relie des entités différenciées ${ }^{14} »$. Qu'il s'agisse des camps de détention insulaires au large de l'Australie ou aux bords méditerranéens de l'Europe, des centres de détention dans les aéroports en Italie, en Belgique, au Portugal, en Irlande ou en France, cette autre frontière que s'avère l'espace de confinement n'est pas un passage horizontal entre deux ordres socio-politiques et deux lieux; plutôt, elle connecte et sépare verticalement deux espaces. A la verticale du réseau frontalier, le maintien s'inscrit en effet dans un autre espace, qui est celui d'un réseau de circulation dans lequel il apparaît paradoxalement comme un enfermement et une transition.

Joseph est arrivé du Congo avec un avion transitant par Istanbul ; il a déchiré son passeport dans l'avion et a demandé l'asile aux policiers français à son arrivée à Roissy. Il a été détenu en zone d'attente à "Zapi 2 », dans une aile du centre de rétention du Mesnil-Amelot, pendant seize jours, au cours desquels sa demande d'asile a été entendue et refusée, puis il a été renvoyé en Turquie. Après plusieurs tentatives de renvoi auxquelles il s'est opposé, Joseph a été escorté jusqu'à Istanbul. Mais les autorités turques ne l'ont pas autorisé à descendre de l'avion et il a été ramené à Paris, enregistré comme "non admis » à nouveau, puis détenu en zone d'attente à «Zapi 3 ", dans le centre d'hébergement de Roissy. Après dix-huit jours de détention, Joseph a été placé en garde-à-vue, jugé en comparution immédiate au tribunal de Bobigny et emprisonné trois mois dans la prison de Fleury-Mérogis, d'où il a adressé sa demande d'asile à l'Office français de protection des réfugiés et apatrides (OFPRA).

Emile est un congolais vivant comme réfugié protégé par le Haut Commissariat pour les réfugiés (HCR) de l'ONU en Côte d'Ivoire, jusqu'à ce qu'il vienne demander l'asile en France suite à des émeutes xénophobes en 2004. Il présente lors de son entretien de demande d'asile plusieurs lettres recommandées qu'il avait envoyées depuis la Côte d'Ivoire au siège du HCR à Genève, pour expliquer ses agressions et demander plus de protection. Le bureau du HCR à Paris confirme son histoire, mais la question est ailleurs: Emile est un réfugié placé sous la convention de l'Organisation de l'unité africaine (OUA) et non

14. Heyman J., op. cit., p. 262. 
celle de Genève ${ }^{15}$. Sa demande d'asile est refusée et il est renvoyé sous escorte à Abidjan.

Li est une jeune femme chinoise arrivée de Pékin en passant par Dakar. "Non admise », elle a passé quelques heures dans le poste de police de l'aéroport de Roissy et a été renvoyée à Dakar dans la journée. Elle est restée trois ans au Sénégal pour rembourser son voyage et racheter un passage : la revoilà contrôlée à la descente de l'avion qui vient de Dakar. Elle est placée une nouvelle fois en zone d'attente à Zapi 3 ; elle se fait désormais comprendre en parlant le wolof.

Oscar et Léon sont deux frères de 12 et 15 ans : ils ont voyagé seuls depuis la Côte d'Ivoire après que des émeutes xénophobes ont décimé leur famille d'origine peule. Leur avion a transité par Pékin, vers où ils sont expulsés après avoir passé quelques jours à Zapi 316.

Souvent, le parcours d'exil qui aboutit en zone d'attente implique un trajet à multiples escales fixées par les passeurs auxquels les voyageurs ont recours. Il n'est pas rare que les demandeurs d'asile aient d'abord demeuré quelques mois, voire quelques années, dans un pays de transit voisin, le temps de renouer les contacts avec la famille, de travailler pour amasser l'argent nécessaire au voyage, etc. Dans ces cas, l'étranger expulsé est renvoyé à une série de déplacements : son expulsion l'enferme dans un exil, dont les limites avec l'errance ne sont pas très claires. Faute de ressources, qu'ils ont déjà mobilisées pour payer leur voyage en avion, voire hypothéquées dans les cas fréquents où l'étranger s'est endetté pour voyager, les individus expulsés sont renvoyés à un transit plus ou moins durable, dans l'espace de la circulation globale. Les nœuds de cet espace sont les aéroports, les routes, les lieux intermédiaires dans lesquels les voyageurs attendent quelques jours leur passeur ou plusieurs années de pouvoir payer le voyage. La zone d'attente s'y inscrit, et si elle a pour ambition de repousser ceux qui ne sont pas autorisés à entrer «de l'autre côté » (comme tout poste de contrôle frontalier), c'est du côté de cette errance-ci qu'elle renvoie les expulsés. Il est dès lors essentiel de creuser les modalités et les enjeux de cet autre enfermement qui se joue en zone d'attente : non plus un enfermement dedans, mais, paradoxalement, un « enfermement dehors », dans un espace de la mobilité contrainte, qui serait une marge intérieure de la globalisation. En effet, cet enfermement dans la circu-

15. Son statut de réfugié en Côte d'Ivoire est ainsi régulé par la «Convention de l'OUA régissant les aspects propres aux problèmes de réfugiés en Afrique » du 20 juin 1974, alors que le statut des demandeurs d'asiles et réfugiés en France est régulé par la «Convention relative au statut des réfugiés » dite « de Genève » du 28 juillet 1951.

16. Les données présentées sont issues d'une enquête de terrain en zone d'attente à Roissy entre fin 2004 et début 2008. Elles ont été recueillies d'une part au cours d'une participation observante dans la zone d'attente et, d'autre part, à partir d'entretiens individuels menés à Paris et en région parisienne avec des personnes ayant eu une expérience de détention frontalière. 
lation participe des modalités contemporaines d'exclusion dans le contexte de la globalisation. En même temps, la zone d'attente est un effet, et même un "réacteur 17 » de ce régime politique et socio-économique, dans la mesure où elle est productrice de nouvelles modalités d'existence.

Les pratiques de contrôle aux frontières se cristallisent pour une part importante autour des usages et du sens attribués au passeport, marqueur d'identité, instrument du monopole étatique des moyens légitimes de circulation ${ }^{18}$ qui situe ce contrôle, parfois de manière problématique, dans un système international de reconnaissance documentaire et de surveillance des frontières. Ainsi, la gestion étatique des déplacements relève d'une gestion des identités, codifiées et institutionnalisées, qui «place les individus dans un espace de surveillance en même temps qu'il les situe dans un réseau d'écriture; il les implique dans une masse de documents qui les capture et les fixe 19 ». Mais qu'en est-il lorsque cette précieuse garantie n'est plus et que le contrôle aux frontières se trouve confronté à une désarticulation du lien entre l'individu en déplacement et l'Etat souverain dont il est le sujet ?

Dharar est un demandeur d'asile somalien dont la demande d'admission sur le territoire au titre de l'asile a été refusée, notamment parce qu'il voyageait avec un visa en règle mais un passeport kenyan falsifié à son nom. Ces documents de voyage ont été confisqués par la police lors de son arrestation à Roissy le 4 mai. Dharar est expulsé une première fois le 13 mai vers le lieu d'escale de son avion: Zurich. La Suisse refuse l'entrée à ce voyageur sans identité, qui est alors renvoyé vers son lieu de provenance, Nairobi, le 14 mai. Le Kenya refuse également de laisser entrer cet homme qu'il ne reconnaît pas comme l'un de ses ressortissants. Un second essai est tenté, et le demandeur d'asile somalien est à nouveau embarqué sur un vol ParisZurich-Nairobi le 15 mai : les pays concernés refusent encore de laisser le voyageur non identifié descendre de l'avion. Durant ces 72 heures, Dharar est resté sous escorte de police, menottes aux poignets, sans s'allonger. Il se retrouve à nouveau maintenu en zone d'attente après avoir parcouru $27113 \mathrm{~km}$ en trois jours.

N'étant pas sorti des zones sous douane durant ce temps, le demandeur d'asile se trouve donc en quelque sorte en orbite autour d'un système international où il n'a littéralement pas de place, son identité n'étant plus inscrite dans le lien à l'Etat-nation qu'institue le passeport. Cette situation - commune en

17. Bayart J-F., op. cit., p. 235.

18. Torpey J., «Aller et venir, le monopole étatique des moyens légitimes de circulation », Cultures E Conflits, $\mathrm{n}^{\circ} 31-32$, automne-hiver 1998, pp. 63-100.

19. Foucault M., Surveiller et punir. Naissance de la prison, Paris, Gallimard, 1975, p. 234. 
zone d'attente - a le nom pittoresque de «ping-pong » dans le jargon policier à la frontière. Lorsque nous interrogeons Dharar sur la raison pour laquelle il n'a pas de passeport somalien, il nous regarde d'un air perplexe avant de répondre que cela fait quinze ans qu'il n'y a plus d'Etat en Somalie. L'étranger qui quitte son pays pour venir en France avec un faux passeport, et qui est refusé sur le territoire, «est libre », selon la loi de 1992, de repartir vers n'importe quelle destination où il sera admis légalement - or, sans passeport, une telle destination ne semble pas exister. Cet exemple vient questionner à la marge le système de monopole étatique de la circulation : l'exceptionnel dans la « mise sur orbite » des voyageurs sans passeport n'est pas la perte d'une place (qui intervient au seuil de chaque migration), mais l'impossibilité d'en retrouver une. La détention aux frontières institutionnalise paradoxalement une errance : il n'y a soudain plus d'endroit sur terre où l'étranger non identifié puisse aller. Ce n'est pas matériellement un problème d'espace (il n'y en aurait plus pour tous) mais «un problème d'organisation politique 20 » dans le système international formé par une communauté d'Etats-nations, quiconque est exclu de l'une des communautés politiques se trouve exclu de la famille des communautés, remarque ainsi Hannah Arendt. Or ce mouvement est à la conjonction des principes de souveraineté qui organisent politiquement le monde contemporain et des principes d'universalisation qui en fondent l'intégration à l'échelle globale : «seule une bumanité complètement organisée pouvait faire que la perte de résidence et de statut politique revienne à être expulsé de l'bumanité entière 21 ». Au-delà de la question du droit d'asile, le réfugié tout comme le voyageur qui déchire son passeport et refuse de donner sa nationalité (l'un se retrouvant sans Etat de jure, l'autre le devenant de facto) témoignent des paradoxes d'une appartenance politique définie doublement, par les propriétés exclusives d'une citoyenneté nationale d'une part, et par les normes universelles de l'ordre international dans lequel s'inscrit la nation, d'autre part. Dans ces "perplexités des droits bumains 22 ", les pratiques de pénalisation de ceux qui restent pris debors apparaissent comme un levier précieux pour régler les modalités d'une exclusion politique et sociale qui cherche à se maintenir dans les cadres juridiques de l'Etat de droit. Ce processus, qui ouvre les frontières en réseaux et en zones habitables, invite plus largement à s'interroger sur le mode d'inclusion différentiel par lequel une conception nationale de la participation politique s'insère dans les normes universelles de citoyenneté dont procède, en théorie, la globalisation.

\section{Techniques de gestion de la circulation}

Dans ce contexte, contrairement à l'idée de flottement insinuée par exemple dans le recours à l'expression de "ping-pong ", les trajets des individus

20. Arendt H., «Le déclin des Etats-nations et la fin des droits de l'Homme », L'Impérialisme, 1981, p. 276.

21. Ibid., p. 282.

22. Ibid. 
soumis à une reconfiguration de la frontière dans ses fonctions et sa matérialité sont influencés, sinon déterminés, par des instruments techniques et juridiques de contrôle, dont nous tenterons de développer quelques traits. En partant des pratiques de refoulement, nous évoquerons ainsi deux dimensions qui sont, d'une part, l'expulsion et ses effets de pouvoir dans le réseau de circulation et, d'autre part, les évolutions de la procédure de maintien dans la perspective d'une gestion des flux.

Pour les demandeurs d'asile à la frontière, la procédure d'examen s'agence autour de l'élaboration d'un récit de vie qui doit convaincre l'officier de protection de l'OFPRA qu'une personne a quitté son pays suite à des « menaces » conformes à l'interprétation française de la Convention de Genève et qu'elle y serait exposée en cas de retour dans ce pays. Ce récit personnel - que les demandeurs d'asile apprennent à construire en intégrant peu à peu ce que signifie l' «asile », quels sont le raisonnement des agents de l'OFPRA et les normes de véracité qui distinguent le mensonge du vraisemblable - exclut une dimension importante du voyage, qui est la façon dont le contrôle à la frontière vient s'inscrire dans l'itinéraire du demandeur d'asile et influence sa trajectoire, notamment en dessinant de nouveaux parcours et en posant de nouveaux enjeux en cas d'expulsion.

Gérard a fui le Congo en 1998 lors des massacres des Rwandais peu après la prise de pouvoir de Laurent Désiré Kabila. Il s’est alors réfugié en Angola. Suite à une série de répressions, après la réélection disputée de Laurent Kabila, Gérard quitte l’Angola au printemps 2007 et, après une escale à Addis-Abeba, il arrive à Roissy où il est détenu durant onze jours. Sa demande d'asile est refusée et il est renvoyé à Addis-Abeba sur un vol de la compagnie Ethiopian Airlines, avec laquelle il avait voyagé à l'aller. A son arrivée, ses documents administratifs sont directement remis à la police de l'aéroport qui le place en détention. En échange de sa libération, l'Etat éthiopien lui demande de rembourser son billet d'avion, ainsi que l'amende payée par le transporteur à la police française. Gérard reste ainsi six mois dans une prison à Addis-Abeba. Quelques mois plus tard, il rentre à Luanda et prend un vol pour Lisbonne. Il se rend ensuite en train jusqu'à Paris, où il attend l'acheminement de certains documents capables de «faire preuve » pour déposer enfin sa demande d'asile.

Dans le cadre de la procédure, un étranger contrôlé aux frontières peut être renvoyé dans son pays d'origine ou vers son lieu de provenance, lequel a souvent été un lieu de transit plus ou moins long. De même, les «menaces » en cas d'expulsion prises en compte par l'officier de protection de l'OFPRA sont celles en rapport avec le récit linéaire d'une mise en danger et d'une fuite, et non celles qui découleront éventuellement de l'expulsion. Au retour, l'étranger 
expulsé est remis aux autorités policières de l'aéroport où il est renvoyé. Bien qu'il soit difficile de documenter ce point, plusieurs témoignages individuels comme ceux de Gérard permettent de réinscrire la détention en zone d'attente dans un parcours plus large d'enfermement et de passage que le contrôle aux frontières et l'expulsion déterminent. Les personnes expulsées sont mises en garde-à-vue dans l'aéroport ou emprisonnées, en raison non pas de l'histoire individuelle qui les a incitées à quitter leur pays, mais d'un délit de départ. Les raisons de l'emprisonnement au retour ne sont pas toujours claires et peuvent être multiples : par exemple, la demande d'une caution coûteuse à un ressortissant assez riche pour entreprendre le voyage en avion vers l'Europe, ou l'assimilation systématique des personnes retournées à des criminels, dans la mesure où les politiques de double peine et de mise en rétention suite à un délit pénal ont institué cette image. Il serait pourtant naif de rester sur cette idée d'arbitraire et de chaos, alors que ces pratiques de double enfermement semblent largement déterminées par les techniques européennes de délocalisation en amont $\mathrm{du}$ contrôle frontalier, à travers notamment deux pratiques : les sanctions contre les transporteurs et la criminalisation de l'émigration.

L'article 26 de la Convention d'application de l'accord de Schengen du 14 juin 1985 engage en effet les Etats parties à instaurer des sanctions contre les transporteurs acheminant des voyageurs considérés comme n’étant pas en règle ${ }^{23}$. Le Conseil des ministres de l'Union européenne a adopté en 2001, suite à une initiative française, une directive ${ }^{24}$ qui complète l'article 26 et tend à harmoniser les sanctions et le dispositif d'obligation des transporteurs. Cette directive fixe des amendes de 3000 à 5000 euros par passager «non admis » et attribue la charge du renvoi aux compagnies de transport. La loi du 26 juillet 1992 indique que les frais de maintien des étrangers en zone d'attente sont à la charge des compagnies aériennes, excepté pour les demandeurs d'asile (admis sur le territoire comme tels après audition à l'OFPRA). Lorsque le transporteur est une compagnie nationale comme Ethiopian Airlines, les autorités peuvent considérer le demandeur d'asile responsable du coût financier, le sanctionner pénalement et lui en demander remboursement.

En outre, une tendance récente du contrôle migratoire en Europe a été l'exportation dans les pays de départ de la notion d' "émigration clandestine ». Cette rhétorique s'est progressivement transformée en gestion pénale d'une nouvelle illégalité, à travers des pratiques plus ou moins institutionnalisées. L'exemple de Gérard montre la punition et l'enfermement de facto des per-

23. Le paradoxe étant que cette appréciation ne dépend pas seulement des documents de voyage (passeport et visa), mais d'autres éléments tels que les certificats d'hébergement, la somme d'argent disponible ou les «motivations » du voyage, dont l'appréciation est du ressort discrétionnaire de la police de l'air et des frontières depuis les circulaires du 8 août et du $17 \mathrm{sep}-$ tembre 1986.

24. Directive 2001/51/CE, Journal officiel L 187 du 10 juillet 2001, pp. 45-46. 
sonnes renvoyées dans les prisons des pays de retour. Le Sénégal a créé une jurisprudence en utilisant la loi de 2005 « relative à la lutte contre la traite des personnes et pratiques assimilées et à la protection des victimes " pour pénaliser des exilés naufragés à des fins de "sanction de l'émigration 25 ». De façon plus nette, le Maroc a adopté une loi contre «l'émigration clandestine ", avec les articles 50 à 52 de la loi 02/03 du 11 novembre 2003. Le contrôle étatique de la circulation, qui se fonde à l'origine sur les règles de franchissement des frontières nationales, se renverse ainsi utilement en une criminalisation du départ.

Une autre dimension du contrôle s'articule autour de la fluidification et de l'accélération du réseau de circulation dans lequel s'inscrit la zone d'attente, dans une logique de gestion en temps réel. Dans la procédure de maintien légalisant la détention aux frontières en 1992, le refus d'entrée en France ne pouvait en principe pas donner lieu à une mesure de renvoi avant l'expiration du délai d'un « jour franc ", soit 24 heures. Cela implique en théorie que les personnes contrôlées par la police dans les postes situés dans les terminaux soient systématiquement transférées dans le centre d'hébergement de la Zapi 3, puisque la procédure fixe un délai de quelques heures après lesquelles il devient illégal de garder les étrangers dans les cellules des postes de police. Depuis la loi du 26 novembre 2003, cette mesure est assouplie. Le « jour franc » n'est pas automatique, mais il doit être demandé par l'étranger. Or en pratique, le formulaire du procès-verbal de non-admission prévoit automatiquement le renoncement à ce droit : il comprend une case « Je veux repartir le plus rapidement possible » sous laquelle il est demandé à l'étranger, arrivé au terme d'un long et coûteux voyage, de signer ${ }^{26}$.

Kushal a demandé l'asile dans un poste de police à son arrivée à l'aéroport de Roissy, mais sa demande n'a pas été enregistrée. Il a été détenu plusieurs heures dans le terminal avant d'être renvoyé à Mangalor. N'ayant pas de passeport, Kushal a passé douze jours en garde-à-vue dans l'aéroport de Mangalor avant d’être renvoyé à Paris. Lors de son arrivé à Roissy, la police l'a mis cette fois sous procédure de « maintien » et l'a transféré dans le centre de Zapi 3.

25. Le Soleil (Dakkar), «Emigration vers l'Espagne : Le capitaine prend un an, les clandestins six mois avec sursis ", 24 août 2006, http://www.lesoleil.sn/article.php3?id_article=11798, consulté le 15 juin 2008.

26. Cette opération doit, selon le texte de loi, se faire dans une langue que l'étranger «comprend ». Dans les faits, la traduction de ces informations diverses (assurées, via un service téléphonique, par un policier parlant la langue ou, plus rarement, un interprète) n'est pas faite : on demande simplement à la personne de signer à l'endroit indiqué. Voir : Anafé, Une France inaccessible. Rapport de visites en aérogares, zone d'attente de Roissy-Charles de Gaulle, décembre 2007. http://www.anafe.org/download/rapports/anafe-rapport-aerogares-dec07.pdf, consulté le 23 septembre 2008 
Le fait que l'étranger puisse « renoncer » au jour franc, comme ce fut le cas de Kushal, ouvre la possibilité de le renvoyer immédiatement. Le droit au jour franc est une des techniques de pouvoir qui organisent le contrôle étatique aux frontières et ses infléchissements dans une logique de gestion des flux. Ses évolutions renvoient à l'institution, en 1992, d'une procédure qui vient légaliser des pratiques administratives dénoncées comme illégales, puis à l'assouplissement de cette procédure en 2003, qui ménage la possibilité de conserver la légalité des pratiques policières (de respecter la " procédure ») tout en créant des conditions d'applications par lesquelles s'annulent ses effets contraignants. Ceci est observable lorsque le demandeur d'asile « renonce» par défaut à ses droits (sur lesquels il ne reçoit pas d'information) lorsqu'il n'en demande pas explicitement l'application. Sous les allures d'une technicité pointilleuse, ces processus s'inscrivent dans un jeu rhétorique de légitimation et d'euphémisation empreint d'une ironie et d'un sens de l'antiphrase certains et, surtout, ouvrent de larges perspectives d'actions policières en matière de renvoi. Ils illustrent ainsi de façon intéressante le jeu entre contrôle étatique et défense juridique, par lequel chacun des acteurs force des marges de manœuvre à l'intérieur du cadre d'action énoncé par l'autre. En 2006, l'exercice du droit à « repartir le plus rapidement possible » a permis de renvoyer immédiatement un tiers (soit $32 \%$ ) des étrangers contrôlés à Roissy : sur un total de 21235 voyageurs «non admis » en France, 14427 personnes ont été maintenues en zone d'attente; quant aux autres, elles «ont demandé à être réacheminées immédiatement » selon le ministère de l'Intérieur ${ }^{27}$. La gestion récente de la détention aux frontières s'inscrit ainsi dans une économie du pouvoir qui cherche à assouplir l'emprise discrétionnaire en l'orientant dans le sens d'une plus grande gestion des flux : ne pas garder les corps dans un espace et une relation de contrôle contestés et coûteux - en capital humain, financier, symbolique - mais les remettre en circulation le plus rapidement possible.

\section{La frontière excluante : un processus social et politique}

La légalisation des pratiques de détention administrative des étrangers a institué plusieurs régimes d'enfermement aux frontières. L'institution des zones d'attente est, avec les centres de rétention et les prisons de droit commun, le troisième régime qui boucle les frontières institutionnelles de la France à travers une détention des étrangers, liée à la transgression des règles pour « le franchissement des frontières et le séjour sur le territoire ». Chacun de ces régimes d'enfermement a ses lieux, ses législations, ses acteurs et sa temporalité. Mais ils forment néanmoins les arêtes d'un dispositif qui maintient les étrangers en circulation dans une marge étanche, qui peut aussi bien être décrite comme une frontière intérieure, un anneau de saturne interne au terri-

27. Compte rendu de la réunion entre l'administration et les organisations sur le fonctionnement des zones d'attente, 16 avril 2007, http://www.anafe.org/stats.php, consulté le 15 juin 2008. 
toire. En partant de la zone d'attente, deux lignes de force permettent de mieux saisir cette circulation séparée. D’un côté, les procédures administratives et juridiques s'articulent entre elles, de sorte qu'elles peuvent faire passer les étrangers d'une situation d'enfermement à une autre. De l'autre, l'introduction des étrangers en procédure administrative dans une procédure de droit commun, à travers la qualification d'illégalismes, permet d'embrayer une succession de situations clandestines, de "procédures prioritaires » et de refus, qui les installent dans une exclusion durable.

Laurent a fui la Côte d'Ivoire et demandé l'asile à son arrivée à Roissy en décembre 2004. A cette époque, la guerre civile pousse des centaines d'ivoiriens à demander l'asile à la frontière, et en face, le ministère de l'Intérieur adopte une politique de refus et de renvois systématiques en Côte d'Ivoire. Laurent résiste à plusieurs tentatives de renvoi : à quelques jours de la fin du délai légal de maintien en zone d'attente (qui est de 20 jours maximum), après une nouvelle résistance à une tentative de renvoi au cours de laquelle Laurent est blessé par la police, il est isolé dans une petite pièce adjacente au poste de police dans le centre fermé puis transféré dans un local de garde-à-vue situé dans l'emprise aéroportuaire. Il est placé en garde-à-vue et est présenté en comparution immédiate au Tribunal correctionnel pour s'être opposé à son renvoi, ce qui l'expose à une sanction pénale pour "infraction d'entrée ou séjour irrégulier et soustraction à l'exécution d'une mesure de refus d'entrée en France ». Sa peine est de 3 mois de prison ferme et trois ans d'interdiction de territoire. A sa sortie de prison, Laurent dépose une demande d'asile à l'OFPRA, qui est très rapidement acceptée : il est reconnu réfugié 2 mois plus tard. Mais lorsque Laurent va à la préfecture pour obtenir sa carte de séjour de 10 ans, celle-ci lui est refusée parce qu'une interdiction de territoire français de 3 ans est enregistrée à son nom sur le registre national. Durant deux ans encore, il vit avec des titres de séjour temporaires qu'il renouvelle tous les 15 jours à 3 mois à la préfecture, puis va s'enregistrer au commissariat de son domicile, est mis sous une procédure d' " assignation à résidence », qui lui demande de ne pas quitter le territoire départemental et de « pointer » au commissariat à intervalles réguliers, jusqu’à ce que son dossier pénal soit «nettoyé » et qu'il puisse obtenir le titre de séjour qui correspond au statut de réfugié qui lui a été reconnu deux ans auparavant.

La lecture de ce parcours donne un goût de l'illisibilité et de la violence des dédales juridico-administratifs dans lesquels sont pris les étrangers contrôlés. Le chevauchement du régime pénal et du régime administratif créé 
un effet de chaos 28 dû à l'absence de concertation entre les différentes instances décisionnelles (les préfectures, l'OFPRA, le Tribunal correctionnel, le Tribunal administratif, etc.) et l'incohérence - voire les contradictions - d'une accumulation de décisions juridiques et administratives. Il en va ainsi de Laurent, que nous avons rencontré par une belle journée d'été dans un studio en demi sous-sol où il passait ses vacances alors qu'il était encore assigné à résidence, réfugié statutaire et interdit de territoire français.

L'espace qui se met en place au croisement de ces procédures est une marge qui accumule de multiples façons - et avec une créativité dont nous n'avons pas le loisir ici d'énumérer toutes les combinaisons - des situations de suspension administrative, d'attente ou de clandestinité et des situations d'enfermement. Un étranger placé en zone d'attente qui résiste à son renvoi forcé peut être pénalisé pour cette raison et enfermé dans une prison de droit commun ${ }^{29}$. A sa sortie de détention, l' « interdiction de territoire » qui a sanctionné son infraction pénale l'expose à une interpellation et un enfermement en centre de rétention administrative. Or, en pratique, une grande partie des étrangers qui sont placés en garde-à-vue à la suite de leur maintien en zone d'attente le sont parce que leur pays d'origine est inconnu et que leur avion de provenance n'a pas été identifié. Face aux modalités d'expulsion qui procèdent par renvoi vers le lieu d'escale ou de provenance de l'avion et par reconnaissance consulaire des nationalités, la résistance des étrangers contrôlés s'organise autour d'une anonymisation. Le premier enjeu est de rendre le trajet aérien illisible en restant assez longtemps dans le terminal de l'aéroport pour que la trace immédiate du vol de provenance soit effacée. Il ne s'agit pas seulement d'une pratique de résistance, mais plutôt d'une réalité ménagée par les conditions même du contrôle, dans la mesure où la police peut refuser d'entendre un demandeur d'asile et le laisser déambuler un certain temps dans les terminaux avant de le garder au poste de police et de le mettre sous procédure de " demande d'asile ». Le second enjeu est celui de la nationalité : ici aussi, la réponse des maintenus aux procédures de renvoi est d'éliminer les traces de nationalité, ce à quoi l'administration répond par le recours à la reconnaissance diplomatique. L'étranger anonyme « de provenance inconnue » est ainsi présenté à l'ambassade du pays dont on le croit originaire, ou encore promené d'ambassade en ambassade si les officiers de police n'ont pas d'idée arrêtée quant à son origine.

28. Voir la partie suivante sur ce point.

29. Ce second enfermement peut sembler paradoxal car il s'oppose à l'accélération des délais de renvoi et de mise en circulation afin d'éviter les coûts dus à l'immobilisation du migrant. Toutefois, les coûts d'enfermement évoqués plus haut concernent la détention frontalière, alors que le passage de l'étranger dans le système pénitentiaire (pour une peine maximale de quatre mois fermes) engage des coûts différents. On pourrait les appeler « utiles », dans le sens où ce passage en prison remplit d'autres fonctions d'illégalisation et de marginalisation dans le parcours de l'étranger, comme nous l'expliquons par la suite. D'autre part, cet enfermement agit surtout là où la mise en circulation est problématique : la garde-à-vue permet de maintenir en situation liminaire les étrangers dont l’anonymat rend le renvoi impossible. 
Youssif a embarqué, depuis une côte africaine, sur une pirogue pourvue d'un moteur de tondeuse à gazon avec une cinquantaine d'autres personnes. Leur embarquement a fait naufrage en Méditerranée et les naufragés ont été repêchés par la flotte militaire française. De Marseille, les quelques survivants ont été conduits à Roissy, en zone d'attente. "De provenance inconnue », Youssif est emmené dans de nombreuses ambassades pour qu'elles le reconnaissent comme leur ressortissant - en vain. Lorsque je le rencontre, ce grand homme sec aux pommettes hautes, au nez fin et à l'accent légèrement arabe a un rendez-vous à l'ambassade de Côte d'Ivoire car il a été surpris à fredonner la chanson ivoirienne que les policiers écoutaient dans le fourgon la veille, au retour d'une ambassade. "Mais la musique ivoirienne, tout le monde l'écoute en Afrique! », précise-t-il.

Cependant, l'identification de l'origine et le jeu des reconnaissances consulaires n'est pas uniquement un enjeu de contrôle et de résistance. Il est plus largement le résultat de cette désarticulation problématique du lien national qui qualifie les réfugiés comme nous l'avons suggéré. En effet, comme en témoignent les pratiques de "ping-pong », quelle ambassade voudra reconnaitre un réfugié palestinien né dans un camp aujourd'hui en Egypte, ou encore, un demandeur d'asile du Cachemire ? Ainsi, la garde-àvue s'impose-t-elle en pratique comme une technique efficace pour maintenir dans le seuil les étrangers dont l'anonymat rend le renvoi impossible, et que la loi impose de libérer au terme des vingt jours de «maintien » en zone d'attente. Mais, de l'autre côté du dispositif frontalier, par-delà la prison et le centre de rétention, il arrive ainsi que l'étranger renvoyé dans un pays, duquel l'administration française a obtenu une reconnaissance consulaire après insistance, soit refusé à la frontière. Le réfugié palestinien expulsé en Egypte est ainsi refusé par les autorités policières du Caire, malgré le complaisant «laisser-passer » finalement délivré par l'ambassade égyptienne à Paris. Détenu plusieurs jours dans l'aéroport du Caire, il est renvoyé en France, contrôlé à Roissy et placé en zone d'attente. La combinaison des procédures administratives et pénales ouvre une marge, où l'intérieur du territoire national communique avec un extérieur - ici l'aéroport du Caire - et dans laquelle les étrangers confinés sont mis en circulation, d'un lieu d'enfermement à un autre. Cette circulation séparée renvoie, au sens propre, à la définition que donnait Michel Foucault de la vie des hommes infâmes : des «vies parallèles $30 »$.

30. Les « vies parallèles» font référence au nom donné par Michel Foucault à la collection dans laquelle il a édité les mémoires d'Herculine Barbin (Herculine Barbin, dite Alexina B., Paris, Gallimard, 1978) et de Pierre Rivière (Moi, Pierre Rivière, ayant égorgé ma mère, ma sæur et mon frère : un cas de parricide au XIXe siècle, Paris, Gallimard, 1973). 


\section{Gestion des illégalismes}

Ces chevauchements et détours administratifs sont pourtant moins chaotiques qu'il n'y parait à première vue. Ce que révèlent en effet les parcours de sortie de zone d'attente, c'est la manière dont la pénalisation de la circulation non autorisée au point d'entrée du territoire met en branle une série d'épreuves juridiques et administratives qui maintiennent l'étranger à distance de son titre de séjour ou de son entretien de demande d'asile - en un mot, agence les conditions d'impossibilité de la régularisation.

Béatrice a fui le Congo lors des violences qui ont suivi les résultats des élections présidentielles en février 2007. Elle est arrivée à Roissy avec un avion de la compagnie Ethiopian Airlines qui transitait par Addis-Abeba. Suite à des irrégularités dans sa procédure de détention, le juge des libertés et de la détention rend une décision mitigée : la détention n'est pas annulée, mais le juge la limite à cinq jours au terme desquels Béatrice devrait être libérée si elle n'est pas renvoyée en Ethiopie ou admise en France comme demandeuse d'asile. Sa demande d'asile est refusée et, à plusieurs reprises, la police la mène à l'aéroport pour la renvoyer vers Addis-Abeba, ce à quoi Béatrice résiste. Au délai du cinquième jour, une équipe policière de l'Unité nationale d'escorte, de soutien et d'intervention (UNESI) vient pour ligoter Béatrice et la mettre dans l'avion. Malgré ses entraves, elle continue cependant à résister au renvoi forcé, de sorte que le commandant de bord annule son expulsion. De retour au poste de police dans le terminal de Roissy, Béatrice est blessée puis emmenée à la prison de Fleury-Mérogis (faute de place dans les locaux de garde-àvue). Elle est jugée en comparution immédiate pour «infraction d'entrée ou séjour irrégulier et soustraction à l'exécution d'une mesure de refus d'entrée en France ». Durant l'audience, Béatrice coupe la parole à son avocat pour se défendre elle-même. Est-ce à cause de ses blessures visibles ou de la cohérence de son récit d'exil ? Le jury ne parvient pas à se mettre d'accord ce jour-là sur sa peine et le prononcé de l'audience est renvoyé à la fin du mois d'août 2007. A sa sortie du tribunal, Béatrice fait une demande d'asile, mais la préfecture refuse de l'enregistrer avant de connâ̂tre le verdict de son jugement, prévu sept mois plus tard. En août 2007, Béatrice est condamnée à deux mois de prison - avec sursis, afin qu'elle puisse déposer sa demande d'asile. Cette fois encore, la préfecture refuse d'enregistrer sa demande d'asile et lui remet sans explication une convocation à la préfecture de police de Paris, au $8^{\mathrm{e}}$ bureau « chargé des mesures d'éloignement des étrangers ». Béatrice se trouve alors dans la situation où le déplacement en préfecture pour enregistrer sa demande d'asile l'expose 
également à une interpellation, puis à une procédure de « rétention ». Elle tente, avec l'assistance juridique de la CIMADE, de communiquer avec le 8 e bureau de la préfecture de police par écrit, sans se présenter physiquement à la convocation. Finalement, en mars 2008, le 8 e bureau autorise Béatrice à déposer sa demande d'asile à la préfecture. La préfecture refuse alors de l'admettre comme demandeuse d'asile et la place sous « procédure prioritaire » de demande d'asile ${ }^{31}$. Le 29 avril 2008, soit un an et deux mois après son arrivée à Roissy, Béatrice est convoquée à la préfecture pour retirer son dossier de demande d'asile, qui sera traité en procédure prioritaire, dans des délais de 96 heures à 15 jours maximum, sans droit de recours suspensif. En attendant, elle ne possède aucun titre de séjour provisoire et se trouve sans-papiers, puisque son admission sur le territoire en tant que demandeuse d'asile a été refusée par la préfecture.

Le refus du statut de «demandeur d'asile » exclut l'étranger des structures d'hébergement, du soutien matériel et du titre de séjour provisoire. Par ailleurs, il le laisse dans une suspension administrative étrange, une absence de décision claire sur sa présence sur le territoire qui ouvre une nouvelle marge discrétionnaire, puisque sa garantie contre les interpellations policières est désormais paradoxalement un « refus d'admission » de la préfecture. Le parcours compliqué de Béatrice révèle comment la pénalisation permet de passer, à l'intérieur même du cadre de l'Etat de droit et de ses dynamiques de pouvoir, d'un régime juridique « normal » à un régime discrétionnaire. La mise à l'écart s'organise en effet à partir d'une interpénétration ambiguë des régimes administratifs et pénaux, qu'illustre l' infraction d'entrée ou séjour irrégulier et soustraction à l'exécution d'une mesure de refus d'entrée en France "sur laquelle se fonde la garde-à-vue. Dès lors, une réflexion sur le régime d'exception ou la suspension $\mathrm{du}$ droit en jeu aux frontières ne peut rentrer dans le grain fin des situations de confinement si elle reste dans les dichotomies du droit et du «non-droit », sans prendre mesure de la façon complexe dont les techniques de gouvernement prennent corps dans des combinaisons entre prérogatives administratives, droit

31. Traitée en quinze jours, cette procédure ne permet pas le « recours suspensif » qui suspend l'expulsion jusqu'à ce qu'une cour ait réétudié, en appel, la demande d'asile refusée par l'OFPRA : le demandeur d'asile refusé est donc expulsable immédiatement, au maximum quinze jours après l'enregistrement de sa demande, ce qui concerne pratiquement tous les étrangers mis sous une procédure d'examen extrêmement mal disposée à leur égard : seuls $2,2 \%$ des demandeurs d'asile sont reconnus réfugiés dans cette procédure, alors que 8,2 \% le sont dans la procédure "normale ", et $26,9 \%$ après un recours devant la Cour nationale du droit d'asile (CNDA) possible dans la procédure normale (d'après l'OFPRA, Rapport d'activité 2005). Ces mauvaises dispositions s'expliquent par les raisons qui motivent la mise sous procédure prioritaire : provenance d'un pays d'origine sûr, demande "manifestement infondée » selon la terminologie de l'OFPRA (c'est-à-dire fausse de toute évidence), ou «troubles à l'ordre public » selon les termes employés par la préfecture de Paris - catégorie dans laquelle entre Béatrice par sa condamnation pénale. 
administratif et droit pénal. En zone d'attente, la législation de 1992 qui institue le « maintien » a fait passer ces pratiques dans le domaine du droit administratif, de même que l'avaient fait celles de 1980 (dite « loi Bonnet ») et de 1981 (dite « loi Peyrefitte ») instituant la « rétention ». Plus particulièrement, ces lois octroient aux agents de la police aux frontières une plus grande liberté de manœuvre par rapport à la procédure pénale de garde-à-vue, laquelle garantit plus de droits aux prévenus que le maintien n'en réserve aux étrangers contrôlés. Elles ouvrent ainsi la possibilité de gestion des flux par enfermement et renvoi forcé, et la facilitent. Cette gestion fondée sur un rapport de pouvoir qui retient et déplace des corps sans cesse et à large échelle, nécessite d'aménager une suspension de facto des prérogatives au-delà de ce qui est admis dans le régime de droit commun - ce que réalisent le maintien ou la rétention en droit administratif. Or, au détour de ces procédures administratives de courte durée s'opère une réinscription du contrôle dans le système pénal : la résistance à l'expulsion expose à la détention ferme et à l’interdit de territoire français.

A travers cette "pénalisation des résistances 32 ", l'infraction et son enregistrement dans le registre national installent alors l'étranger contrôlé dans un statut de délinquant qui détermine l'ensemble de son parcours administratif à venir : à la préfecture, à l'OFPRA, au tribunal, à l'hôpital, dans un commissariat, etc. L'observation empirique suggère que l'effet de pouvoir recherché par cette gestion de la circulation en illégalisme n'est pas tant l'enfermement de l'étranger que le recensement et la catégorisation opérées via l'enregistrement d'une sanction pénale. La pénalisation légitime et rend techniquement possible une mise à l'écart administrative de long terme, en désactivant par avance les possibilités ultérieures de régularisation ou de participation politique de l'étranger mis au ban. Dans ce contexte, les personnes mises à l'écart doivent, pour négocier éventuellement leur inclusion, s'exposer à des situations où le pouvoir administratif a emprise sur leur personne. C'est ce que montre la Circulaire ministérielle "relative aux conditions de l'interpellation d'un étranger en situation irrégulière, garde-à-vue de l'étranger en situation irrégulière, réponses pénales» du 21 février $2006{ }^{33}$. Au regard de l'encadrement légal des conditions du contrôle d'identité ${ }^{34}$, la circulaire précise qu' "est licite le contrôle d'identité d'étrangers occupant sans titre un bâtiment, en l'espèce une église, et revendiquant publiquement l'irrégularité de leur situation administrative ». D'autre part, la circulaire détaille le mécanisme d'arrestation des étrangers sans papiers convoqués par la préfecture ou qui y viennent de leur propre

32. Foucault, M., « La société punitive », Dits et Ecrits, II, Paris, Gallimard, 1994, p. 467.

33. Circulaire du 21 février 2006 de Pascal Clément et Nicolas Sarkozy, parue au Bulletin officiel du ministère de la Justice, $\mathrm{n}^{\circ} 101,1^{\mathrm{er}}$ janvier-31 mars 2006.

34 . En principe, un contrôle d'identité ne devrait pas pouvoir se faire n'importe où : une interpellation «déplacée » annule la procédure administrative de détention de l'étranger contrôlé. La circulaire du 21 février 2006 insiste, au sujet des interpellations, sur «le respect des exigences procédurales qui en garantiront la régularité. La mise en oeuvre de ce type d'interpellation conditionne souvent, en effet, l'effectivité de la mesure de reconduite à la frontière ». 
gré pour régulariser leur situation. L'emprise physique du pouvoir sur les individus cible donc précisément les situations administratives ou politiques qui cherchent à négocier une possibilité de régularisation - d'inclusion dans la communauté nationale - dans un registre politique « normal » (la manifestation ou le rendez-vous à la préfecture pour retirer un formulaire). Elle pointe par là la fonction de frontière de ces interactions politiques et administratives, en renvoyant à une définition originelle de la frontière comme le lieu de l'exposition des sujets à la manifestation du pouvoir ${ }^{35}$. Par là, ces pratiques d'interpellation participent du dispositif de mise en en circulation dans l'enfermement dans lequel s'inscrit la zone d'attente.

\section{Enfermement et contrôle : les données du problème}

La volonté de criminalisation de l'étranger met à jour deux dimensions qui nous amènent à réévaluer les techniques de gouvernement et les processus politiques à l'œuvre aux frontières, pour en apprécier les nouvelles modalités de mise en pratique. D'une part, le droit n'est à nul moment suspendu lors de cette mise en circulation paradoxale, et il semble au contraire que des réajustements de la procédure soient continuellement effectués. Cette procédure est une pratique, sans cesse perfectionnée dans le sens d'une inclusion étanche dans l'Etat de droit. Dans ce contexte, le discrétionnaire ne semble ni un débordement accidentel, ni une « décision » souveraine, mais une technique de gestion à flux tendu - lorsqu'il s'agit d'enfermer ou de déplacer les corps - qui ouvre ses marges de manœuvre dans les vides ou les superpositions contradictoires d'un enchevêtrement de régimes juridiques et administratifs. D'autre part, le dispositif frontalier dans lequel est pris l'étranger contrôlé en zone d'attente n'agit pas seulement en immobilisant et en enfermant des personnes, détenues littéralement dans la frontière, mais il opère plus largement dans le temps et l'espace par une recension et une catégorisation qui organisent une mise à l'écart au long cours. Ces processus d'exclusion constituent les contours d'une logique de contrôle des flux, dont les effets se superposent à la privation de liberté. Face à cette dernière, les initiatives de défense des droits fondamentaux contre la détention administrative ont permis de réinscrire les prérogatives souveraines dans un jeu serré d'avancées du pouvoir et de résistances du droit. Reste, de la même façon, à formuler les mécanismes du contrôle des flux dans un questionnement politique et social à même d'énoncer les possibilités d'une résistance.

35. "This construction of the literal border as a state-space where sovereign laws, but not state rights, are applied is reminiscent of the Middle-ages [...] at the borders; travellers are subject to the unlimited power of the state.", in Salter M., Rights of Passage: the Passport in International Relations, Boulder, Lynne Rienner, 2003, p. 127. 